Tohoku Math. J.

56 (2004), 491-499

\title{
ON THE NONEXISTENCE OF STABLE CURRENTS IN SUBMANIFOLDS OF A EUCLIDEAN SPACE
}

\author{
XUESHAN ZHANG
}

(Received February 25, 2003, revised November 11, 2003)

\begin{abstract}
In 1973, Lawson and Simons conjectured that there are no stable currents in any compact, simply connected Riemannian manifold $M^{m}$ which is $1 / 4$-pinched. In this paper, we regard $M^{m}$ as a submanifold immersed in a Euclidean space and prove the conjecture under some pinched conditions about the sectional curvatures and the principal curvatures of $M^{m}$. We also show that there is no stable $p$-current in a submanifold of $M^{m}$ and the $p$-th homology group vanishes when the shape operator of the submanifold satisfies certain conditions.
\end{abstract}

1. Introduction. For any compact Riemannian manifold $M^{m}$, a theorem of Federer and Fleming [1] asserts that any non-trivial integral homology class in $H_{p}(M, Z)$ corresponds to a stable current. By using techniques from the calculus of variations in geometric measure theory together with the Federer-Fleming theorem, Lawson and Simons [3] showed that there are no stable currents in a sphere $S^{m}$, and there is no stable $p$-current in submanifolds immersed in $S^{m}$ with sufficiently small second fundamental form. The result of [3] on submanifolds of $S^{m}$ has been extended to submanifolds of a Euclidean space $E^{m}$ or of $S^{m_{1}} \times S^{m_{2}}$ by Xin [4] and Zhang [5], respectively.

In [3], Lawson and Simons conjectured that there are no stable varifolds, in particular, there are no stable currents in any compact, simply connected Riemannian manifold $M^{m}$ which is 1/4-pinched. As variants of the above conjecture, there have been many results on stable minimal submanifolds and stable harmonic maps. However, the original conjecture by Lawson and Simons is still open. In 1985, with comparison theorems, Howard [2] proved that there is a constant $\delta(m, p)>1 / 4$ such that there is no stable $p$-current in $M^{m}$ when $M^{m}$ is $\delta(m, p)$-pinched. However, the expression of $\delta(m, p)$ is complex and unfortunately $\lim _{m \rightarrow \infty} \delta(m, p)=1$.

In this paper, we regard such a compact Riemannian manifold $M^{m}$ as a submanifold immersed in a Euclidean space $E^{m+k}$ with essential codimension $k$. We shall prove that there are no stable currents in $M^{m}$ if one of the following conditions is satisfied:

1) The sectional curvatures $K_{M}>(k / 4)\left(\lambda_{0}-\mu_{0}\right)^{2}$, where $\lambda_{0}$ and $\mu_{0}$ are the maximum and the minimum of the principal curvatures of $M^{m}$, respectively;

2) $\lambda \mu>(1 / 4)(\lambda-\mu)^{2}$, where $\lambda$ and $\mu$ are any two principal curvatures of $M^{m}$.

The condition 1) indicates that Lawson-Simons' conjecture is true if $k\left(\lambda_{0}-\mu_{0}\right)^{2} \leq 1$ on $M^{m}$.

2000 Mathematics Subject Classification. Primary 53C20; Secondary 53C40, 49Q15.

Key words and phrases. Stable current, submanifold, shape operator, sectional curvature. 
With condition 2), we can prove that if any two principal curvatures $\lambda, \mu$ of a compact submanifold $M^{m}(m>3)$ in $E^{m+k}$ satisfy the inequality $3-2 \sqrt{2}<\lambda / \mu<3+2 \sqrt{2}$, then $M^{m}$ is homeomorphic to a sphere.

Let $N^{n}$ be a compact submanifold immersed in the manifold $M^{m}$ satisfying the above condition 1) or 2) and $S$ a $p$-rectifiable set in $N^{n}$. By using the shape operators $A_{\lambda}$ associated with the immersion $N^{n} \rightarrow M^{m}$, we introduce a selfadjoint linear operator $Q^{A}$ on $p$-space $T_{x} S \subset T_{x} N$. We shall show that there is no stable $p$-current in $N^{n}$ if, for any $x \in N^{n}$ and any $p$-subspace $V$ in $T_{x} N, \operatorname{tr} Q^{A} \leq 0$.

2. Preliminaries. We use the same notation as in $[3,5]$ throughout this paper. In this section, we recall several formulas employed in [5]. Also, we prove three lemmas which will be used in the proof of our main theorems.

Let $M^{m}$ be an $m$-dimensional compact Riemannian manifold with metric $\langle$,$\rangle and Levi-$ Civita connection $\nabla$. Denote by $(S, \xi)$ an oriented, $p$-rectifiable set in $M^{m}$. The set of rectifiable $p$-currents is defined by

$$
\Re_{p}(M)=\left\{\mathcal{S}=\sum_{n=1}^{\infty} n \mathcal{S}_{n} \mid \mathcal{S}_{n}=\left(S_{n}, \xi_{n}\right), M(\mathcal{S})=\sum_{n=1}^{\infty} n \mathcal{H}^{p}\left(S_{n}\right)<\infty\right\} .
$$

$\mathcal{S} \in \Re_{p}(M)$ is called an integral $p$-current if $\mathcal{S}$ and the boundary $\partial \mathcal{S}$ are both rectifiable currents. The space of integral $p$-currents is denoted by $\mathfrak{s}_{p}(M)$. The direct sum $\mathfrak{\Im}_{*}(M)=$ $\bigoplus_{p} \mathfrak{I}_{p}(M)$ together with the boundary operator $\partial: \mathfrak{I}_{*}(M) \rightarrow \mathfrak{I}_{*}(M)$ forms a differential chain complex.

FEDERER-Fleming THEOREM [1]. For each $p \geq 0$ there is a natural isomorphism

$$
H_{p}\left(\mathfrak{I}_{*}(M)\right) \cong H_{p}(M, Z) .
$$

Moreover, for each $\alpha \in H_{p}\left(\mathfrak{I}_{*}(M)\right)$ there exists a current $\mathcal{S} \in \alpha$ of "least mass", that is, $M(\mathcal{S}) \leq M\left(\mathcal{S}^{\prime}\right)$ for all $\mathcal{S}^{\prime} \in \alpha$.

Let $\phi_{t}: M^{m} \rightarrow M^{m}$ be a 1-parameter group of local diffeomorphisms generated by a smooth vector field $X \in C(T M)$. A current $\mathcal{S} \in \Re_{p}(M)$ is said to be stable if for each $X \in C(T M)$ there is an $\varepsilon>0$ such that

$$
M\left(\phi_{t} * \mathcal{S}\right) \geq M(\mathcal{S})
$$

for all $|t|<\varepsilon$. This implies that for any $X \in C(T M)$ we have

$$
\left.\frac{d}{d t} M\left(\phi_{t^{*}} \mathcal{S}\right)\right|_{t=0}=0,\left.\quad \frac{d^{2}}{d t^{2}} M\left(\phi_{t^{*}} \mathcal{S}\right)\right|_{t=0} \geq 0 \text {. }
$$

Let $(S, \xi)$ be an oriented, $p$-rectifiable set. Then $\xi$ is an $\mathcal{H}^{p}$-measurable section of $\bigwedge^{p} T M$ over $S$ with the property that for $\mathcal{H}^{p}$-almost all $x \in S, \xi_{x}$ is a simple $p$-vector of unit length representing $T_{x} S$. For such a pair $(S, \xi)$, let

$$
Q_{\xi}(X)=\left.\frac{d^{2}}{d t^{2}}\left\|\phi_{t^{*}} \xi\right\|\right|_{t=0} .
$$


Then the second variation formula derived by Lawson and Simons [3] can be given by

$$
\left.\frac{d^{2}}{d t^{2}} M\left(\phi_{t^{*}} \mathcal{S}\right)\right|_{t=0}=\sum_{n} n \int_{S_{n}} Q_{\xi_{n}}(X) d \mathcal{H}^{p}(x) .
$$

If $X=\nabla f$ for some $f \in C^{3}(M)$, and $\left\{e_{i}, e_{\alpha}\right\}(i=1,2, \ldots, p ; \alpha=p+1, \ldots, m)$ is an orthonormal basis of $T_{x} M$ with $\xi=e_{1} \wedge \cdots \wedge e_{p}$, then (see [3, p. 436])

$$
Q_{\xi}(X)=\left[\sum_{i}\left\langle a^{X}\left(e_{i}\right), e_{i}\right\rangle\right]^{2}+2 \sum_{i, \alpha}\left\langle a^{X}\left(e_{i}\right), e_{\alpha}\right\rangle^{2}+\sum_{i}\left\langle\nabla_{X, e_{i}} X, e_{i}\right\rangle,
$$

where $a^{X}\left(e_{i}\right)=\nabla_{e_{i}} X$ and $\nabla_{X, e_{i}} X=\nabla_{X} \nabla_{e_{i}} X-\nabla_{\nabla_{X} e_{i}} X$.

For a $p$-rectifiable set $S$ in $M^{m}$, it is known that for $\mathcal{H}^{p}$-almost all points $x \in S$, there exists an approximate $p$-space $T_{x} S \subset T_{x} M$, to $S$. Now we introduce an operator $Q^{A}$ on $T_{x} S$ and establish the relationship between $Q^{A}$ and $Q_{\xi}$.

Let $\varphi: M^{m} \rightarrow E^{m+k}$ be an isometric immersion of $M^{m}$ into the Euclidean $(n+k)$-space $E^{m+k}$ and $D$ the Levi-Civita connection of $E^{m+k}$. Denote by $V\left(E^{m+k}, M\right)$ the normal bundle of $M^{m}$ in $E^{m+k}$. For a smooth section $v \in C\left(V\left(E^{m+k}, M\right)\right)$, let $A_{v}$ be the shape operator determined by $v$. At $x \in M^{m}, A_{v}: T_{x} M \rightarrow T_{x} M$ is a selfadjoint linear operator, which is defined by

$$
A_{v} X=-\left(D_{X} v\right)^{T},
$$

where $X \in C(T M)$. Let $\left\{v_{\lambda}\right\}$ be an orthonormal basis of the normal space $V_{x}\left(E^{m+k}, M\right)$ and $A_{\lambda}=A_{v_{\lambda}}$. For a given integer $p \in(0, m)$, let $V$ be a $p$-dimensional subspace in $T_{x} M$ and $\left\{e_{i}\right\}$ an orthonormal basis of $V$. Define a selfadjoint linear map $Q^{A}: V \rightarrow V$ associated with the immersion $\varphi$ by

$$
Q^{A} X=\sum_{\lambda}\left[2\left(\sum_{i}\left\langle A_{\lambda}^{2} X, e_{i}\right\rangle e_{i}-B_{\lambda}^{2} X\right)-\left(\operatorname{tr} A_{\lambda}-\operatorname{tr} B_{\lambda}\right) B_{\lambda} X\right],
$$

where $X \in V$ and $B_{\lambda}$ is a map on $V$ associated with $A_{\lambda}$ defined by

$$
B_{\lambda} X=\sum_{i}\left\langle A_{\lambda} X, e_{i}\right\rangle e_{i}
$$

$Q^{A}$ is independent of the choice of bases of $V_{x}\left(E^{m+k}, M\right)$ and $V$. Its trace is given by

$$
\operatorname{tr} Q^{A}=\sum_{i}\left\langle Q^{A} e_{i}, e_{i}\right\rangle=\sum_{\lambda}\left[2 \sum_{i, \alpha}\left\langle A_{\lambda} e_{i}, e_{\alpha}\right\rangle^{2}-\left(\operatorname{tr} A_{\lambda}-\operatorname{tr} B_{\lambda}\right) \operatorname{tr} B_{\lambda}\right],
$$

where $\left\{e_{\alpha}\right\}$ is an orthonormal basis of $V^{\perp}$ which is the orthogonal complement of $V$ in $T_{x} M$.

Let $(S, \xi)$ be an oriented, $p$-rectifiable set. At $x \in S$, we associate a tangent $p$-space $V=T_{x} S \subset T_{x} M$. Choose an orthonormal basis $\left\{e_{i}, e_{\alpha}\right\}$ of $T_{x} M$ such that $\left\{e_{i}\right\}$ is a basis of $V$ and $\xi=e_{1} \wedge \cdots \wedge e_{p}$. We now consider $Q_{\xi}$ given by (2.2) as a quadratic form defined on the set

$$
\theta=\left\{v^{T} \in C(T M) \mid v \in E^{m+k}, v^{T}(x)=\text { orthogonal projection of } v \text { onto } T_{x} M\right\} .
$$


Note that at $x \in M^{m},\left\{e_{i}, e_{\alpha}, v_{\lambda}\right\}$ is an orthonormal basis of $E^{m+k}$. Therefore,

$$
\operatorname{tr} Q_{\xi}=\sum_{i} Q_{\xi}\left(e_{i}\right)+\sum_{\alpha} Q_{\xi}\left(e_{\alpha}\right)+\sum_{\lambda} Q_{\xi}\left(v_{\lambda}\right) .
$$

Making use of the proof given in [5], we obtain

LEMMA 1 [5]. Let $M^{m}$ be a submanifold immersed in $E^{m+k}$. Then

$$
\operatorname{tr} Q_{\xi}=\operatorname{tr} Q^{A} \text {. }
$$

LEMMA 2. Let $x_{1}, x_{2}, \ldots, x_{m} ; y_{1}, y_{2}, \ldots, y_{m}$ be real numbers satisfying

$$
\sum_{a}\left(x_{a}\right)^{2}=\sum_{a}\left(y_{a}\right)^{2}=1, \quad \sum_{a} x_{a} y_{a}=0 .
$$

Then for given real numbers $k_{1}, k_{2}, \ldots, k_{m}$, we have

$$
\left(\sum_{a} k_{a} x_{a} y_{a}\right)^{2} \leq \frac{1}{4}\left(k_{\max }-k_{\min }\right)^{2},
$$

where $k_{\max }=\max _{1 \leq a \leq m}\left\{k_{a}\right\}, k_{\min }=\min _{1 \leq a \leq m}\left\{k_{a}\right\}$.

PROOF. In order to get the maximum of the function $f=\left(\sum_{a} k_{a} x_{a} y_{a}\right)^{2}$ under the constraints (2.7), partially differentiating

$$
F=\left(\sum_{a} k_{a} x_{a} y_{a}\right)^{2}+C_{1}\left[\sum_{a}\left(x_{a}\right)^{2}-1\right]+C_{2}\left[\sum_{a}\left(y_{a}\right)^{2}-1\right]+C_{3} \sum_{a} x_{a} y_{a}
$$

with respect to each variable and equating to zero, we obtain

$$
\begin{aligned}
& 2 k_{a} y_{a} \sum_{b} k_{b} x_{b} y_{b}+2 C_{1} x_{a}+C_{3} y_{a}=0, \\
& 2 k_{a} x_{a} \sum_{b} k_{b} x_{b} y_{b}+2 C_{2} y_{a}+C_{3} x_{a}=0,
\end{aligned}
$$

where $C_{1}, C_{2}, C_{3}$ are Lagrange multipliers. Set $u=\sum_{a} k_{a}\left(x_{a}\right)^{2}, v=\sum_{a} k_{a}\left(y_{a}\right)^{2}, w=$ $\sum_{a} k_{a} x_{a} y_{a}$. Then the above equations can be rewritten as

$$
\begin{aligned}
& 2 w k_{a} y_{a}+2 C_{1} x_{a}+C_{3} y_{a}=0, \\
& 2 w k_{a} x_{a}+2 C_{2} y_{a}+C_{3} x_{a}=0 .
\end{aligned}
$$

By using the constraints (2.7), from (2.8) and (2.9) we have

$$
2 w^{2}+2 C_{1}=0, \quad 2 w v+C_{3}=0 ; \quad 2 w u+C_{3}=0, \quad 2 w^{2}+2 C_{2}=0 .
$$

Hence,

$$
C_{1}=C_{2}=-w^{2}=-f, \quad C_{3}=-2 w v=-2 w u .
$$

If $w=0$, then $f=0$. 
Now assume that $w \neq 0$. Then $C_{1}=C_{2} \neq 0$, and $C_{3}=-2 w v=-2 w u$ gives $u=v$. From (2.8) and (2.9) we obtain

$$
2 C_{1}\left[\left(x_{a}\right)^{2}-\left(y_{a}\right)^{2}\right]=0 .
$$

Hence, $\left(x_{a}\right)^{2}-\left(y_{a}\right)^{2}=0, a=1,2, \ldots, m$. Without loss of generality, we may suppose

$$
x_{s}=y_{s}(s=1, \ldots, k) ; \quad x_{t}=-y_{t}(t=k+1, \ldots, m) .
$$

Then (2.8) or (2.9) implies

$$
\begin{gathered}
\left(2 w k_{s}+2 C_{1}+C_{3}\right) x_{s}=0 \quad(s=1, \ldots, k), \\
\left(2 w k_{t}-2 C_{1}+C_{3}\right) x_{t}=0 \quad(t=k+1, \ldots, m) .
\end{gathered}
$$

Now, $\sum_{a} x_{a} y_{a}=\sum_{s}\left(x_{s}\right)^{2}-\sum_{t}\left(x_{t}\right)^{2}$; from (2.7) we have $\sum_{s}\left(x_{s}\right)^{2}=\sum_{t}\left(x_{t}\right)^{2}=1 / 2$. Hence there are at least an $s_{0}$ and a $t_{0}$ such that $x_{s_{0}} \neq 0$ and $x_{t_{0}} \neq 0$. Therefore we obtain

$$
2 w k_{s_{0}}+2 C_{1}+C_{3}=0, \quad 2 w k_{t_{0}}-2 C_{1}+C_{3}=0 .
$$

From (2.11) and (2.10) we get

$$
k_{s_{0}}=-\frac{2 C_{1}+C_{3}}{2 w}=w+u, \quad k_{t_{0}}=\frac{2 C_{1}-C_{3}}{2 w}=u-w,
$$

which imply that $w=(1 / 2)\left(k_{s_{0}}-k_{t_{0}}\right)$. Consequently, $f=w^{2} \leq(1 / 4)\left(k_{\max }-k_{\min }\right)^{2}$.

A similar argument proves the following

LEMMA 3. Let $x_{1}, x_{2}, \ldots, x_{m} ; y_{1}, y_{2}, \ldots, y_{m}$ be real numbers satisfying

$$
\sum_{a}\left(x_{a}\right)^{2}=\sum_{a}\left(y_{a}\right)^{2}=1, \quad \sum_{a} x_{a} y_{a}=0 .
$$

If given real numbers $k_{1}, k_{2}, \ldots, k_{m}$ satisfy

$$
k_{a} k_{b}>\frac{1}{4}\left(k_{a}-k_{b}\right)^{2}(\text { resp., } \geq), \quad a, b=1,2, \ldots, m,
$$

then

$$
2\left(\sum_{a} k_{a} x_{a} y_{a}\right)^{2}-\sum_{a} k_{a}\left(x_{a}\right)^{2} \sum_{b} k_{b}\left(y_{b}\right)^{2}<0(\text { resp., } \leq) \text {. }
$$

3. Main results. In this section, we assume that $M^{m}$ is an immersed submanifold in $E^{m+k}$ with essential codimension $k$, i.e., $M^{m}$ can be immersed in $E^{m+k}$, but not in $E^{m+l}$ with $l<k$.

Let $\left\{v_{\lambda}\right\}$ be an orthonormal basis of the normal space $V_{x}\left(E^{m+k}, M\right)$, and associate the shape operator $A_{\lambda}=A_{v_{\lambda}}$. From the Gauss equation in $M^{m}$, the sectional curvature of the 2-plane $X \wedge Y \subset T_{X} M$ is

$$
K(X \wedge Y)=\sum_{\lambda}\left[\left\langle A_{\lambda} X, X\right\rangle\left\langle A_{\lambda} Y, Y\right\rangle-\left\langle A_{\lambda} X, Y\right\rangle^{2}\right]
$$

where $X$ and $Y$ are mutually orthogonal unit vectors in $T_{x} M$. 
THEOREM 1. Let $M^{m}$ be a compact submanifold in $E^{m+k}$. Denote by $\lambda_{0}$ and $\mu_{0}$ the maximum and the minimum of the principal curvatures of $M^{m}$, respectively. If the sectional curvatures of $M^{m}$ satisfy

$$
K_{M}>\frac{k}{4}\left(\lambda_{0}-\mu_{0}\right)^{2}
$$

then there are no stable currents in $M^{m}$ and $H_{p}(M, Z)=0$ for any $p \in(0, m)$.

PRoOF. For each fixed index $\lambda$, there is an orthonormal basis $\left\{E_{a}\right\}$ in $T_{x} M$ such that

$$
A_{\lambda} E_{a}=k_{\lambda a} E_{a}, \quad a=1,2, \ldots, m .
$$

Let $(S, \xi)$ be an oriented $p$-rectifiable set in $M^{m}$. With a point $x \in S$, associate a tangent $p$-space $V=T_{x} S \subset T_{x} M$. Choose an orthonormal basis $\left\{e_{i}, e_{\alpha}\right\}$ of $T_{x} M$ such that $\left\{e_{i}\right\}$ is a basis of $V$. Also, let

$$
e_{i}=\sum_{a} e_{i}^{a} E_{a}, \quad e_{\alpha}=\sum_{a} e_{\alpha}^{a} E_{a}
$$

Then

$$
\sum_{a}\left(e_{i}^{a}\right)^{2}=1, \quad \sum_{a}\left(e_{\alpha}^{a}\right)^{2}=1, \quad \sum_{a} e_{i}^{a} e_{\alpha}^{a}=0
$$

and

$$
A_{\lambda} e_{i}=\sum_{a} e_{i}^{a} k_{\lambda a} E_{a}
$$

Hence we have

$$
\left\langle A_{\lambda} e_{i}, e_{\alpha}\right\rangle=\sum_{a} e_{i}^{a} e_{\alpha}^{a} k_{\lambda a} .
$$

From Lemma 2 we see that $f=\left(\sum_{a} e_{i}^{a} e_{\alpha}^{a} k_{\lambda a}\right)^{2}$, under the constraints (3.5), has the maximum $(1 / 4)\left(k_{\max }-k_{\min }\right)^{2}$, where $k_{\max }=\max _{1 \leq a \leq m}\left\{k_{\lambda a}\right\}, k_{\min }=\min _{1 \leq a \leq m}\left\{k_{\lambda a}\right\}$. Therefore,

$$
\left\langle A_{\lambda} e_{i}, e_{\alpha}\right\rangle^{2} \leq \frac{1}{4}\left(\lambda_{0}-\mu_{0}\right)^{2} .
$$

In (3.6), $\lambda_{0}$ and $\mu_{0}$ are the maximum and the minimum of the principal curvatures of $M^{m}$, respectively, which are independent of the index $\lambda$.

Combining (3.1) and (2.5), we obtain

$$
\operatorname{tr} Q^{A}=-\sum_{i, \alpha} K\left(e_{i} \wedge e_{\alpha}\right)+\sum_{\lambda, i, \alpha}\left\langle A_{\lambda} e_{i}, e_{\alpha}\right\rangle^{2} .
$$

From (3.6) and (3.7), the condition (3.2) implies tr $Q^{A}<0$.

Let $\theta$ be the set given by (2.6). If $v^{T} \in \theta, v^{T}$ is the gradient $\nabla f$ of the function $f(x)=$ $\langle v, x\rangle$ on $M^{m}$. For $X \in \theta$, let $\phi_{t}$ be the flow generated by $X$ and set

$$
Q_{\mathcal{S}}(X)=\left.\frac{d^{2}}{d t^{2}} M\left(\phi_{t^{*}} \mathcal{S}\right)\right|_{t=0},
$$


where $\mathcal{S} \in \Re_{p}(M)$. Then from (2.1) we have

$$
\operatorname{tr} Q_{\mathcal{S}}=\sum_{n} n \int_{S_{n}} \operatorname{tr} Q_{\xi_{n}} d \mathcal{H}^{p}(x)
$$

From Lemma 1, we get $\operatorname{tr} Q_{\xi_{n}}=\operatorname{tr} Q^{A}<0$. Hence, $\operatorname{tr} Q_{\mathcal{S}}<0$. This implies that there is no stable $p$-current in $M^{m}$ for any $p \in(0, m)$. Federer-Fleming's theorem then shows that $H_{p}(M, Z)=0, p=1,2, \ldots, m-1$.

A similar argument as in Theorem 1 proves the following

THEOREM 2. Let $M^{m}$ be a compact submanifold immersed in a simply connected space form $N^{m+k}(c)$ with $c \geq 0$. Denote by $\lambda_{0}$ and $\mu_{0}$ the maximum and the minimum of the principal curvatures of $M^{m}$, respectively. If the sectional curvatures of $M^{m}$ satisfy

$$
K_{M}>\frac{k}{4}\left(\lambda_{0}-\mu_{0}\right)^{2}-c
$$

then there are no stable currents in $M^{m}$ and $H_{p}(M, Z)=0$ for $1 \leq p \leq m-1$.

THEOREM 3. Let $M^{m}$ be a compact submanifold immersed in $E^{m+k}$. If any two principal curvatures $\lambda, \mu$ of $M^{m}$ satisfy

$$
\lambda \mu>\frac{1}{4}(\lambda-\mu)^{2},
$$

then there are no stable currents in $M^{m}$ and $H_{p}(M, Z)=0$ for $p=1,2, \ldots, m-1$.

Proof. As in the proof of Theorem 1, for each fixed index $\lambda$, from (3.3) and (3.4) we have

$$
A_{\lambda} e_{i}=\sum_{a} e_{i}^{a} k_{\lambda a} E_{a}, \quad A_{\lambda} e_{\alpha}=\sum_{a} e_{\alpha}^{a} k_{\lambda a} E_{a}
$$

where $e_{i}^{a}$ and $e_{\alpha}^{a}$ satisfy the conditions (3.5). Hence we have

$$
\begin{gathered}
\left\langle A_{\lambda} e_{i}, e_{\alpha}\right\rangle=\sum_{a} e_{i}^{a} e_{\alpha}^{a} k_{\lambda a}, \\
\operatorname{tr} A_{\lambda}=\sum_{i}\left\langle A_{\lambda} e_{i}, e_{i}\right\rangle+\sum_{\alpha}\left\langle A_{\lambda} e_{\alpha}, e_{\alpha}\right\rangle=\sum_{i, a}\left(e_{i}^{a}\right)^{2} k_{\lambda a}+\sum_{\alpha, a}\left(e_{\alpha}^{a}\right)^{2} k_{\lambda a}, \\
\operatorname{tr} B_{\lambda}=\sum_{i}\left\langle A_{\lambda} e_{i}, e_{i}\right\rangle=\sum_{i, a}\left(e_{i}^{a}\right)^{2} k_{\lambda a} .
\end{gathered}
$$

Therefore

$$
\begin{aligned}
2 \sum_{i, \alpha}\left\langle A_{\lambda} e_{i}, e_{\alpha}\right\rangle^{2}-\left(\operatorname{tr} A_{\lambda}-\operatorname{tr} B_{\lambda}\right) \operatorname{tr} B_{\lambda} \\
\quad=\sum_{i, \alpha}\left[2\left(\sum_{a} e_{i}^{a} e_{\alpha}^{a} k_{\lambda a}\right)^{2}-\sum_{a}\left(e_{i}^{a}\right)^{2} k_{\lambda a} \sum_{b}\left(e_{\alpha}^{b}\right)^{2} k_{\lambda b}\right] .
\end{aligned}
$$


From Lemma 3 and the assumption of Theorem 3, (3.9) then implies that

$$
2 \sum_{i, \alpha}\left\langle A_{\lambda} e_{i}, e_{\alpha}\right\rangle^{2}-\left(\operatorname{tr} A_{\lambda}-\operatorname{tr} B_{\lambda}\right) \operatorname{tr} B_{\lambda}<0 .
$$

Hence, from Lemma 1, we obtain $\operatorname{tr} Q_{\xi_{n}}=\operatorname{tr} Q^{A}<0$. Consequently, from (3.8), we have $\operatorname{tr} Q_{\mathcal{S}}<0$.

REMARK 1. The inequality $\lambda \mu>(1 / 4)(\lambda-\mu)^{2}$ is equivalent to

$$
[\lambda-(3-2 \sqrt{2}) \mu][\lambda-(3+2 \sqrt{2}) \mu]<0,
$$

which is also equivalent to

$$
3-2 \sqrt{2}<\frac{\lambda}{\mu}<3+2 \sqrt{2} .
$$

By the same proof as used for the second part of Theorem 1 in [6], we can prove the following

THEOREM 4. Let $M^{m}(m>3)$ be a compact connected and orientable submanifold immersed in $E^{m+k}$. If any two principal curvatures $\lambda, \mu$ of $M^{m}$ satisfy the inequality (3.10), then $M^{m}$ is homeomorphic to a sphere.

THEOREM 5. Let $M^{m}$ be a submanifold in $E^{m+k}$. Suppose that its principal curvatures satisfy one of the following conditions:

C1. $\lambda \mu \geq(1 / 4)(\lambda-\mu)^{2}$,

C2. the sectional curvatures $K_{M} \geq(k / 4)\left(\lambda_{0}-\mu_{0}\right)^{2}$,

where $\lambda_{0}$ and $\mu_{0}$ are the maximum and the minimum of the principal curvatures of $M^{m}$, respectively. Let $N^{n}$ be a compact submanifold immersed in $M^{m}$ and $p \in(0, n)$ a given integer. If, for any $x \in N^{n}$ and any $p$-subspace $V$ in $T_{x} N$, the selfadjoint map $Q^{A}$ on $V$ associated with the immersion $N^{n} \rightarrow M^{m}$ satisfies

$$
\operatorname{tr} Q^{A}<0
$$

then there is no stable p-current in $N^{n}$ and $H_{p}(N, Z)=H_{n-p}(N, Z)=0$.

Proof. Let $\nabla, \bar{\nabla}, D$ be the Levi-Civita connections of $N^{n}, M^{m}, E^{m+k}$, respectively. Associated with the immersion $N^{n} \rightarrow E^{m+k}$, the shape operator $A_{v}^{\prime}$ determined by $v \in$ $C\left(V\left(E^{m+k}, N\right)\right)$ is given by

$$
A_{v}^{\prime} Y=-\left(D_{Y} v\right)^{T}
$$

where $Y \in C(T N)$. In particular, if $v \in C(V(M, N))$,

$$
A_{v}^{\prime} Y=-\left(D_{Y} v\right)^{T}=-\left[\bar{\nabla}_{Y} v+\bar{h}(v, Y)\right]^{T}=-\left(-A_{v} Y+\nabla_{Y}^{\perp} v\right)^{T}=A_{v} Y,
$$

where $\bar{h}$ is the second fundamental form of the immersion $M^{m} \rightarrow E^{m+k}$. Also, if $v \in$ $C\left(V\left(E^{m+k}, M\right)\right)$, then

$$
A_{\nu}^{\prime} Y=-\left(-\bar{A}_{\nu} Y+\bar{\nabla}_{Y}^{\perp} v\right)^{T}=\left(\bar{A}_{\nu} Y\right)^{T} .
$$

At $x \in N^{n}$, we take an orthonormal basis $\left\{v_{\lambda}, \eta_{a}\right\}$ of $V_{x}\left(E^{m+k}, N\right)$ so that $\left\{v_{\lambda}\right\}$ and $\left\{\eta_{a}\right\}$ are bases of $V_{x}(M, N)$ and $V_{x}\left(E^{m+k}, M\right)$, respectively. Let $A_{\lambda}=A_{v_{\lambda}}$ and $\bar{A}_{a}=\bar{A}_{\eta_{a}}$. Denote by 
$Q^{A^{\prime}}$ the selfadjoint linear operator on $V$ associated with the immersion $N^{n} \rightarrow E^{m+k}$ defined by (2.3). It follows from (3.11) and (3.12) that

$$
\operatorname{tr} Q^{A^{\prime}}=\operatorname{tr} Q^{A}+\bar{A}(V),
$$

where

$$
\bar{A}(V)=\sum_{a, i, \alpha}\left[2\left\langle\bar{A}_{a} e_{i}, e_{\alpha}\right\rangle^{2}-\left\langle\bar{A}_{a} e_{\alpha}, e_{\alpha}\right\rangle\left\langle\bar{A}_{a} e_{i}, e_{i}\right\rangle\right] .
$$

By using Lemma 3 with the condition $\mathrm{C} 1$, or Lemma 2 with $\mathrm{C} 2$, we then obtain $\bar{A}(V) \leq$ 0. If $\operatorname{tr} Q^{A}<0$, then (3.13) implies $\operatorname{tr} Q^{A^{\prime}}<0$, and from (3.8), $\operatorname{tr} Q_{\mathcal{S}}<0$. The proof is completed.

REMARK 2. If $M^{m}=E^{m}$, then $K_{M}=\lambda_{0}=\mu_{0}=0$ in $E^{m}$, and Theorem 4 reduces to Theorem 1 of Xin [4].

\section{REFERENCES}

[ 1 ] H. Federer And W. Fleming, Normal and integral currents, Ann. of Math. 72 (1960), 458-520.

[2] R. HOWARD, The nonexistence of stable submanifolds, varifolds, and harmonic maps in sufficiently pinched simply connected Riemannian manifolds, Michigan Math. J. 32 (1985), 321-334.

[ 3 ] H. B. LAWSON AND J. Simons, On stable currents and their application to global problems in real and complex geometry, Ann. of Math. 98 (1973), 427-450.

[ 4 ] Y. L. XIN, An application of integral currents to the vanishing theorems, Sci. Sinica Ser. A 27 (1984), 233241.

[ 5 ] X. S. ZHANG, Nonexistence of stable currents in submanifolds of a product of two spheres, Bull. Austral. Math. Soc. 44 (1991), 325-336.

[6] X. S. ZHANG, Geometry and topology of submanifolds immersed in space forms and ellipsoids, Kodai Math. J. 17 (1994), 262-272.

DEPARTMENT OF MATHEMATICS

SHANGHAi UNIVERSITY OF ENGINEERING SCIENCE

XIANXIA ROAD 350

SHANGHAI 200336

THE PEOPlE's REPUBLiC OF CHINA

E-mail address: zhangx_s@citiz.net 